\begin{tabular}{|c|c|c|c|c|c|c|}
\hline & 总 & $\begin{array}{c}\text { Bezeichnung } \\
\text { des Elementes }\end{array}$ & 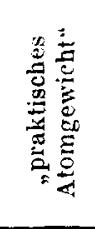 & & 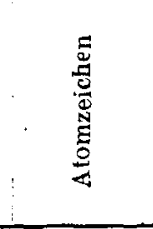 & 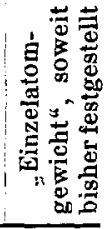 \\
\hline & $E m$ & Emanation . & 222 & $\begin{array}{l}\text { Radium-Emana- } \\
\text { fion }\end{array}$ & $R a E m$ & 222 \\
\hline & - & - & & & & \\
\hline & $R a$ & Radium & 226.0 & $\begin{array}{l}\text { Aktinium } X \\
\text { Thorium } X \\
\text { Radium } \\
\text { Mesothorium } 1\end{array}$ & $\begin{array}{l}A c X \\
T h X \\
R a \\
M s T h_{1}\end{array}$ & $\begin{array}{l}(222) \\
224 \\
226.0 \\
228\end{array}$ \\
\hline & $A c$ & Aktinium. & & $\begin{array}{l}\text { Aktinium } \\
\text { Mesothorium } 2\end{array}$ & $\begin{array}{l}\text { Ac } \\
M S T h_{2}\end{array}$ & $\begin{array}{c}(226) \\
228\end{array}$ \\
\hline & $T h$ & Thorium & 232.1 & 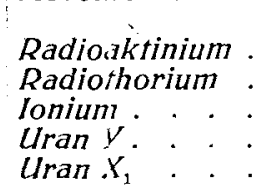 & $\begin{array}{l}\text { RaAc } \\
\operatorname{RaTh} \\
\operatorname{lo} \\
U y \\
U X_{1}\end{array}$ & $\left(\begin{array}{l}(226) \\
228 \\
\left.230^{2}\right) \\
(230) \\
234\end{array}\right.$ \\
\hline & $P a$ & Protaktinium & & $\begin{array}{c}\text { Protaktinium } \\
\text { Uran } X_{2} \\
\qquad \text { (Brevium) }\end{array}$ & $\begin{array}{c}P a \\
U X_{2}(B v)\end{array}$ & $\mid \begin{array}{l}(230) \\
234\end{array}$ \\
\hline & $u$ & Uran & 236.2 & $\begin{array}{l}\text { Uran II } \\
\text { Uran I. }\end{array}$ & $\begin{array}{l}U I I \\
U I\end{array}$ & $\begin{array}{l}234 \\
238\end{array}$ \\
\hline
\end{tabular}

Die Einzel- $A$ tomgewichte sind mit der Genauigkeit angegeben, wie sie die Bestimmungsmethode zuläß31 (in den besten Fällen etwa $1\left(1, m_{0}\right)$. Da diese Methoden also vorläufig nicht die Genauigkeit beanspruchen können, mit der die praktischen Atomgewichte festgestellt werden konnten, so ist es nicht zu entscheiden, ob die sicher vorhiandenen Abweichun ren von der Ganzzahligkeit, wie z. B. beim Stickstcff $(14,01)$ oder beim Phosphor $(31,04)$ auf spurenweise Beimengungen von lsotopen oder aul Massendefekte zurückzuführen sind.

Das Weitere ergibt sich aus den Anmerkungen, die der Tabelle II beigefügt sind

M. Bodenstein, O. Hahn,

O. Hönigschmid, R. J. Meyer, W. Ostwald, Vors.

\section{Das Madruckverfahren und seine Bedeutung für die chemische Industrie.}

Von Berg. und Hütteningenieur Heinrich CARo-Berlin.

(Eingeg. 24.18. 1921.)

Aus dem Ruhrrevier kommen beunruhigende Nachrichten ïber die Aussichten der Winterversorgung des inländischen Steinkohlenbedarfs, bei der alles in allem drei Momente ungünstig dabei ins Gewicht fallen: 1. der fortdauernde Mangel an Qualitätskohlen; 2. der bevorstelıende Wagenmangel und 3. der Rückgang der Lagerbestände, der sich in der nichsten Zeit besonders unangenehm bemerkbar machen wird. Dadurch wird die wichtige Nebenprodukterzeugung erheblich in Mitleidenschatt gezogen werden und ihre Rückwirkung auf die chemische Industrie wird besonders stark hervortreten. Der Ausfall an Nebenprodukten wiirde sich allerdings teilweise aufbalancieren lassen, wenn maln die in der Torttrockensubstanz enthaltenen wertvollen Nebenprodukte, der chemischen Industrie zuführen könnte.

Bei der Entrasung des Torfs mit 60 oder $40 \% \mathrm{H}_{2} \mathrm{O}$ ergeben sich außer dem hochwertigen Torfkoks, der für die Feineisenindustrie von hohem Wert ist, Leuchtgas, leichte und schwere Ole, Paraffin, Essigsäure. Holzgeist, Amınoniak, Asphalt, Butter-, Valerian- und Metacetonsäure, während die Vergasung des Torfs neben Kraftgas den wertvollen Stickstoff in liorm von Ammoniak fördert. Eine Fülle wertvoller, wissenschaf tlicher Arbeit ist auf diesem Gebiet geleistet worden, und es sei hier besonders auf die Arbeiten von Hoering, Petten. kofer, Thenius, Wa grenmann, Vohl und vornehmlich auf die klassischen Arbeiten von N. Caro und Frank hingewiesen.

Die Grundlage einer wirtschaftlichen Erzeugung der Nebenprodukte wird immer die Beschaffung eines Halbtrockenguts mit $60-40^{\circ} \%$ H.O sein. Die Lufttrocknung, deren man sich bis jetzt bedienen mußte, bietet aber wegen ihrer Abhängigkeit von der Witterung und der kurzen Trocknungsperiode von etwa 100 Tagen keine Gewähr für einen zuverlässigen großindustriellen Betrieb. Man muß daher die 'orfowinnung linabhängig von Witterung und Saison machen; und das kann nur dadurch geschehen, das3 dem Torf sein gewaltiger

1) Der Wert wurd durch direkte Dicht --Bestimmung innerhalb der Versuchsfehler bestätigt.

¿) Der Wert wurde durch experimentelle Atomgewichts-Bestimmung eines Ionium-Thoriun-Gemisches gestütrt.
Wasserballast durch mechanische Abpressung entzogen wird. Aber dies läßt sich nur ermöglichen, wenn vor der Abpressung der kolloidale Charakter des Torfs beeinflußt oder zerstört worden ist.

Zerselzter Rohtorf ist als eine kolloidale Lösung anzusprechen, in dem sich neben Resten noch guterhaltener Zellmembranstoffe Zersetzungsprodukte bilden, die das eigenartige Verhalten des Torfes beim Entwässern und Trocknen bedingen. In einer kolloidalen Lösung sind die Humusteilchen außerordentlich fein verteilt, nicht wie $z$ B. zwischen Sandkörnern und Wasser im nassen Sandboden, sondern wie gequollene Gelatine. Daraus resultiert die starke Anziehungskraft zwischen dem Wasser und der Trockensubstanz, und dies bedingt wiederum die Unmöglichkeit, lediglich durch Pressung das Wasser von der Trockensubstanz abzuspalten. Will man also das Wasser abspalten, so muß zunächst die Oberflächenspannung gestört werden, d. h. die Haftspannung, die das Wasser in der Trockensubstanz festhäl, aufgehoben werden.

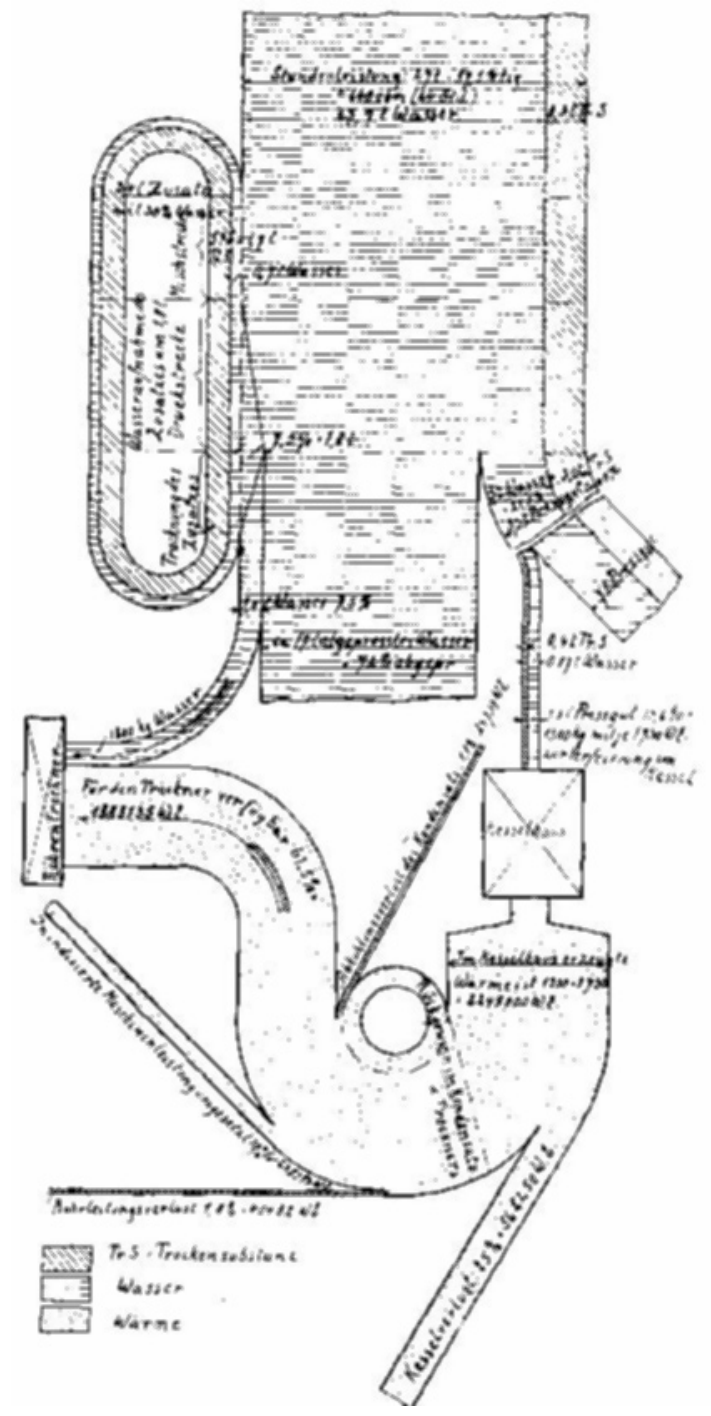

Mehrere Wege führen zu diesem Ziel. Den einfachsten Weg ging Alexanderson in Stockholm, der das Torfkolloid durch Frost zerstörte und die aufgetaute Masse abpreßte. Das Preßgut läßt sich aber wegen seiner pulverigen Beschaffenheit nicht verwerten. Das Verfahren des Grafen Schwerin-Wildenboff und der Elektro-PlatCoal-Werke in Kilberry, Irland, das sogenannte Elektro-Osmose-Verfahren, wendet den elektrischen Strom zur Abspaltung des kolloidal gebundenen Wassers an, während Eckenberg und ten-Bosch überhitzten Dampf aufwenden; aber der Wirkungsurad ist bei dem hohen Eigenbedarf dieser Verfahren zu gering, um eine Wirtschaftlichkeit $\mathrm{zu}$ erzielen.

Einen neuen Weg zur Iösung des Problems eröffnet das Verfahren der Gesellschaft für maschinelle Druckentwässerung in Ürdingen a. Niederrhein, das unter der Bezeichnung "Madruckverfahren " als zurzeit wirtschaftlichstes Verfahren in den Vordergrund getreten ist. Das Verfahren, das von den Dipl.-Ing. Brune und Horst ausgearbeitet wurde, ist von der Technischen Abteilung für Torfwirtschaft bei der Landesanstalt für Moorwirtschaft in München während des letzten Jahres eingehend geprüft worden. Die Technische Abteilung hatte neuerdings die Prüfungsergebnisse auf der Ausstellung für Wasserkraft und Energiewirtschaft in München ausgestellt, und man kann sagen, daß durch dies Verfahren der langgesuchte Weg gefunden und das Problem, Torf lediglich durch Druck zu entwässern, gelठ̋st erscheint. 
Das Madruckverfahren basiert auf der Tatsache, daß das Torfkolloid beeinflußt werden kann, wenn ein fein verteilter Zusatzstof dem Rohtorf beigemengt wird, der eine Verănderung der Oberflächen. spannung des kapillar festgehaltenen Wassers und eine Herabsetzung des Quellungsdruckes des Torfkolloids bewirkt. Als Zusatz verwendet man den Stoff, den man an Ort und Stelle zur Verfügung hat, nämlich Trockentorf mit $30 \% \mathrm{H}_{2} \mathrm{O}$, der bezogen auf die im Rohmoor enthaltene Trockensubstanz im Verhältnis von $1: 2$ beigemengt wird. Dadurch gelingt es, Halbtrockengut von $50-60 \%, \mathrm{H}_{2} \mathrm{O}$ bei einem Eigenbedarf von $20 \%$ des Produktes; also mit einem Wirkungsgrad von $80 \%$ zu erzeugen.

Der Beweis fur diese Angabe soll an der Hand eines praktischen Preßvorganges im nachfolgénden geliefert werden.

Das gebaggerte Rohmoor wird zunächst mittels einer Feldbahn zu einer Torfvorratsgrube gebracht, um den Betrieb dreischichtig durchzufübren, während die Baggerung einschichtig erfolgt. Aus der Vorratsgrube wird der Torf durch einen Elevator mit Hilfe eines Transportbandes in Reißwolfe befördert, die den Torf ohne - und dies ist sehr wesentlich für die Durchfihhrung des Prozesses - ihn zu quetschen oder zu kneten, wodurch eine Strukturveränderung vermieden wird, in kleinkörnige Stücke zerreißen. Der zerkleinerte Torf fällt auf ein Transportband, das mit einer Schicht $30 \% /{ }_{0}$ igem Trockentorf bedeckt ist, und wandert in eine Mischtrommel, wo die Substanzen lose miteinander vermengt werden. Das Mischgut wird nun durch einen Elevator auf ein Transportband und Rücklauftransporteur gebracht, der die Ringpresse beschickt, die das Mischgut auf $60 \%$ und darunter abprefst. Durch die Abpressung wird dem Rohmoor vier Fünftel seines Wasserballastes entzogen, so daß das Preßgut sich fast trocken anfühlt und sieben läßt. Von diesem Preßgut wird nun ein Teil abgetrennt, der mittels eines Transportbandes nach dem Trockner gebracht und auf $30 \%$ herunter getrocknet wird, um von neuem als Trockenzusatz dem Rohmoor beigegeben den Kreislauf zu schließen (s. Abb.) Als Schulbeispiel soll nur ein Rohmoor mit 87,5\% $\mathrm{H}_{2} \mathrm{O}$ angenommen werden und eine Madruckanlage, die $640 \mathrm{cbm}$ pro Tag verarbeitet. Diese Zahlen sind aus dem Grunde angenommen, weil ein Torffeld von $1 \mathrm{qkm}$ und einer Mächtigkeit von $3 \mathrm{~m}$ bei der angenommenen Förderung etwa 15. Jahre ausreicht. Auf die Arbeitsstunde berechnet, werden $27 \mathrm{t}$ dem Preßprozeß unterworfen. Da, wie oben ausgeführt, der Trockenzusatz zu der im Rohmoor befindlichen Trockensubstanz im Verhältnis von $1: 2$ steht, so beträgt die Zusatzmenge 1,7 $\mathrm{t} 30 \%$ iges Torfpulver, die sich beim Preßprozeß auf etwa $60 \% \mathrm{H}_{2} \mathrm{O}$ anreichert, also $\mathrm{um}$ etwa $1,8 \mathrm{t}$ auf $3,5 \mathrm{t}$. Diese $1,8 \mathrm{t} \mathrm{H}_{2} \mathrm{O}$ müssen, damit ein $30^{\circ}$ iges Zusatzgut für den Kreislauf des Prozesses zurückgewonnen wird, im Röhrentrockner verdampft werden, der durch den Abdampf der Betriebsmaschine geheizt wird. Dem aus der Presse austretenden $60^{\circ}$ igen Prefgut wird die pro Stunde zur Verfeuerung notwendige Menge entnommen, in diesem Falle $1,3 \mathrm{t}=15,6^{\circ} / \mathrm{o}$, während $7 \mathrm{t}=84,4^{0}$ zur weiteren Verwendung bleiben.

Zur Erzeugung des gesamten Kraftbedarfs und der benötigten Wärmemenge für den Trockner ist eine Dampfanlage von 300 PSi erforderlich, die, wenn der auf $300^{\circ}$ überhitzte Zudampf eine Spannung von 12 Atm. und der zum Trockner überströmende Abdampf eine solche von $2 \mathrm{Atm}$. besitzt, $8,6 \mathrm{~kg}$ Dampf pro PSi/St. verbraucht. Nimmt man den Wirkungsgrad der Kesselanlage zu $75 \%$ an und ferner, da13 die Spannung im Kessel $12,5 \mathrm{Atm}$. Úberdruck bei $330^{\circ}$ Überhitzung beträgt, und daß das Kondensat aus dem Trockner mit $100^{\circ}$ abfliebt und mit $90^{\circ}$ " wieder in den Kessel gelangt, so beträgt bei einem stündlichen Dampfverbrauch von $8,6 \times 300=2580 \mathrm{~kg}$ die Erzeugungswärme pro $\mathrm{kg}$ Dampf $=655 \mathrm{WE}$. und die stündlich aufzuwendende Wärmemenge $=2249000$ WE. Der hierzu notwendige Brennstoff wird dem aus der Presse austretenden $60 \%$ igen Preßgut entnommen. Da $1 \mathrm{~kg}$ dieses Materials bei $5 \%$ Aschengehalt etwa 1730 W.E. besitzt, so sind hiervon pro Stunde $1300 \mathrm{~kg}$ erforderlich, das sind $15,6^{\prime \prime},(=1,3 \mathrm{t})$ der Gesamtmenge des Preßgutes.

Für die erzielten $1730>1300=2249000$ W.E. ergibt sich nun folgende theoretische Wärmeverteilung:

Kesselverlust

Rohrleitungsverlust

in indizierte Maschinenleistg. umgesetzt 238394

für den Trockner verfügrbar

Abkühlıngsverlust des Kondensats . $\quad 24739$

Summa 2219400 WE. St. $=100^{\circ}$

Eine Störung der Oberflächenspannung durch Teilchen von Trockentorf zeigt bereits im Mischgut Verinderungen durch die Annahme einer flockigen und krümeligen Beschaffenheit. Die disperierten Teilchen verdichten sich auf der Oberfläche des Zusatzkörpers, z nächst noch getrennt durch Flüssigkeitshäutchen stärkerer Konzeatration. Nach Ost wald wird durch diese Aggregation die Bildung eines Hydrogels bewirkt infolge der nunmehr eintretenden weiteren Anniiheiung durch den Preßdruck. Zugleich kann das nicht mehr gebundene Wasser auf den durch die Pressung gebildeten Schichten ablaufen. Nunmehr ist das Humusteilchen zum Dispersionsmittel geworden und Wasser zur dispergierten Phase, wobei in diesem Zustand ein kleiner Teil bei der Auflösung in Wasser sich noch fein verteilt und eintretende Krustenbildung den Vorgang verlangsamt. Dipl.-Ing. Groß von der 'lechnischen Abteilung für Torfwirtschaft in München stellt null anf Grund seiner eingehenden Versuche, die teils in Mïnchen im Laboratorium der bodenkundlichen Abteilung der Forst- lichen Versuchsanstalt mittels eines Madruck-Prebelementes und dann mittels der großen. Versuchsanlage der Gesellschaft in Úrdingen ausgeführt wurden, folgende physikalische Merkmale im Hinblick auf das Madruckverfahren auf: Die Humusstoffe verlieren bei zunehmender Zersetzung das kapillare Aufsaugevermőgen und erhöhen die molekulare Anziehungskraft zu Wasser, wobei sie sich zu einer Teilchengroße von $10^{-8}$, den sogenannten Amikronen entwickeln und sich dadurch der Molekulargröße von 10-7 nähern. Sie besitzen infolgedessen starke innere Reibung, so daß sich Rohtorf mit uber $75 \%$ Wassergebalt noch formen und zerkleinern läßt. Sie zeigen elektronegatives Verhalten und besitzen geringere Leitfähigkeit als Goldlösung, die als typischer Vertreter der Kornchenkolloide angesehen werden kann und setzen der Fällung durch Elektrolyse ziemlich starken Widerstand entgegen. Die Reversibilität in den frtheren Zustand reicht bis $\mathrm{zu}$ einem gewissen Wassergehalt. Getrockneter Torf zeigt eine irreversible Zustandsänderung. Die Adsorptionsfähigkeit der Humusstoffe ist ăußerst stark entwickelt. Dieser Punkt ist in bezug auf das $\mathrm{Ma}$ druckverfahren ganz besonders hervorzuheben, da durch die Einlagerung von fein gemahlenem Torf in die Rohmasse Oberflächenwirkungen eintreten, die für die jeweilige Abgabe von Wasser von bestimmender Bedeutung sind.

Das Preßgut, das in festen Kuchen der Presse entfällt, hat seinen Moorcharakter vollkommen verloren und ähnelt der Rohbraunkohle in so hohem Maße, daß man füglich sagen kann, eine Madruckanlage stellt eine Braunkohlengrube dar. Wie Rohbraunkohle läßt sich das Preßgut auf Treppenrosten verfeuern und läßt sich vortrefflich brikettieren. Die bayerische Landeskohlenstelle gibt über die aus dem nach dem Madruckverfahren entwässerten Moor hergestellten Briketts folgendes Gutachten: Die Briketts mit $15 \%$ Wassergehalt haben einen unteren Heizwert von $4400-4500$ WE. und einen oberen Heizwert von $4130-4570 \mathrm{WE}$. Aschengehalt auf Trockensubstanz bezogen, bei Hochmooren von $0,5-3$, höchstens $5 \%$, bei abbauwürdigem guten Niederungsmoor 5-10\% Die Festigkeit der Briketts ist sehr gut, besser als bei Braunkohlenbriketts. Sie sind nicht selbstentzündlich und nahezu schwefelfrei. Verhalten im Feuer: Das Brikett behält die Form und ist langflammig, seine Asche ist gutartig. Die Briketts sind reinlich und schmutzen nicht.

Ganz besonders eignet sich das Preßgut wegen seiner porösen Beschaffenheit zur Vergasung und Entgasung und eröffnet somit, wie oben erwähnt, der deutschen chemischen Industrie eine neue Quelle, aus der sie die wertvollen Nebenprodukte schöpfen kann. [A. 202.]

\section{Aus der Technik. \\ Neuartige elektrische Öfen für Temperaturen von 2500 Grad $C$ und darüber.}

In Göttingen haben sich die altbekannten Firmen der Feinmechanik, Optik und Elektrotechnik zu der, Verkaufsvereinigung Götinger Werkstätten, G. m. b. H. Göttingen, Geiststr. 3, zusammengeschlossen, um ihre Apparate gemeinsam durch diese Verkaufsvereinigung vertreiben zu lassen, um ferner Ausstellungen gemeinsam zu beschicken und so ein Bild zu geben von den Leistungen der Göttinger Industrie. - Das Göttinger Elektro-Schaltwerk, welches dieser Vereinigung angehört, stellte zur Ausstellung anläßlich des Physikertages in Jena einen nenen elektrischen Ofen aus, welcher sich besonders durch die hohen Temperaturen, die man mit demselben schnelI erreichen kann, ferner durch die äußierst feine Regulierbarkeit der Temperaturen, durch sehr geringen Stromverbrauch, große Sauberkeit, große Konstanz der Temperatur, bequeme Handhabung und leichte Transportfähigkeit auszeichnet.

Mit Hilfe des Ofens ist es möglich, Temperaturen bis $2500^{\circ} \mathrm{C}$ und noch höher zu erreichen und alle Temperaturgrade in den feinsten Grenzen einzustellen, was für sehr viele chemische, physikalische $\mathrm{u}$. a. Versuche von grőßter Wichtigkeit ist. - Das Prinzip entspricht ungefähr demjenigen der bekannten Schmelzeinrichtungen nach NernstTam mann.

In den primären Stromkreis eines Einphasen-Wechselstroms ist ein Schiebewiderstand geschaltet, welcher gestattet, die primäre Spannung und Stromstärke in den allerfeinsten Grenzen zu regulieren. An die sekundäre Seite von großer Stromstärke wird dann der Ofen unter Zwischenschaltung eines Ampèremeters angeschlossen. - Die jeweilige Spannung des Ofens kann an einem Voltmeter abgelesen werden.

Die ganze Anlage ist sehr kompendiös gehalten (siehe Abb.). Jer Transformator ist in einem perforierten Kasten untergebracht. Die Meßinstrumente, Schalter, Sicherungen, Widerstände versenkt gebaut, der Ofen ist auf dem perforierten Kasten kippbar angeordnet.

Die Öfen bestehen im wesentlichen aus den Heizröhren oder Muffeln, welche direkt vom Sirom durchflossen werden. Dieselben sind umgeben von Mantel und Isoliermaterial. Die Rohre und Muffeln sind sebr leicht auswechselbar; je nach ihrer Wandstärke konnen verschiedene Höchsttemperaturen erreicht werden. - Der Ofen ist in jeder Lage benutzbar und kippbar, so dal3 das Schmelzgut bequem ausgegossen werden kann. - Da die Wärmeabgabe der Heizrohre, resp. der Heizmuffeln nach innen eine sehr gute, nach außen aber sehr gering ist, ist der Wirkungsgrad des Ofens sehr günstig. 